Sir,

\section{Lightning-induced cataract}

The first case of lightning-induced cataract was reported in 1722 by St Yves. ${ }^{1}$ Cataracts are the most frequently noted intraocular lesions in lightning strike victims, being typically posterior subcapsular and arising 1 month to 2 years after the injury. ${ }^{2}$ To our knowledge this is the first reported case of lightning-induced cataract in the UK.

\section{Case report}

A 25-year-old healthy woman was struck by a bolt of lightning while walking on a seaside cliff. Her boyfriend, who was with her at the time, was killed instantly. She was admitted to hospital the same day, and on examination was found to have third degree burns to her scalp, the right side of her forehead and all her toes. Routine investigations were normal apart from a markedly elevated creatine kinase (811 IU/l) and urinary myoglobin $(800 \mu \mathrm{g} / \mathrm{l})$.

Subsequent examination by a maxillofacial surgeon revealed a pulpitis of the left upper premolar and molar teeth secondary to vigorous clenching during the strike.

Four days after the injury she presented to the eye department with discomfort in the right eye.

Examination revealed a visual acuity of $6 / 9+2$ in the right eye and $6 / 5$ in the left eye. The right eye was injected and the cornea showed fine punctate epithelial erosions inferiorly. The anterior chamber was quiet and the intraocular pressure was $18 \mathrm{mmHg}$. The disc, vessels and macula appeared normal. She was treated with chloramphenicol ointment.

Further management by the plastic surgeons included amputation of the right fourth toe and debridement of the others, as well as excision of the full thickness scalp burns.

Two months later her visual acuity was $6 / 18$ in the right eye and $6 / 5$ in the left eye. Slit-lamp examination showed a right posterior subcapsular cataract (Fig. 1). Soon afterwards she underwent right

phacoemulsification and intraocular lens implant. Postoperatively the visual acuity was $6 / 5$ and there were no apparent retinal or other sequelae of her injury.

\section{Discussion}

Lightning-induced ocular injury can be caused by several different mechanisms. On average a $2 \mathrm{~km}$ lightning bolt discharges 50-100 million volts ${ }^{2}$ and can produce tissue damage by direct electrolysis, resistance-induced heat or mechanical disruption by the associated shock waves. ${ }^{3}$ According to Ohm's law, current flow between any two points in a circuit is

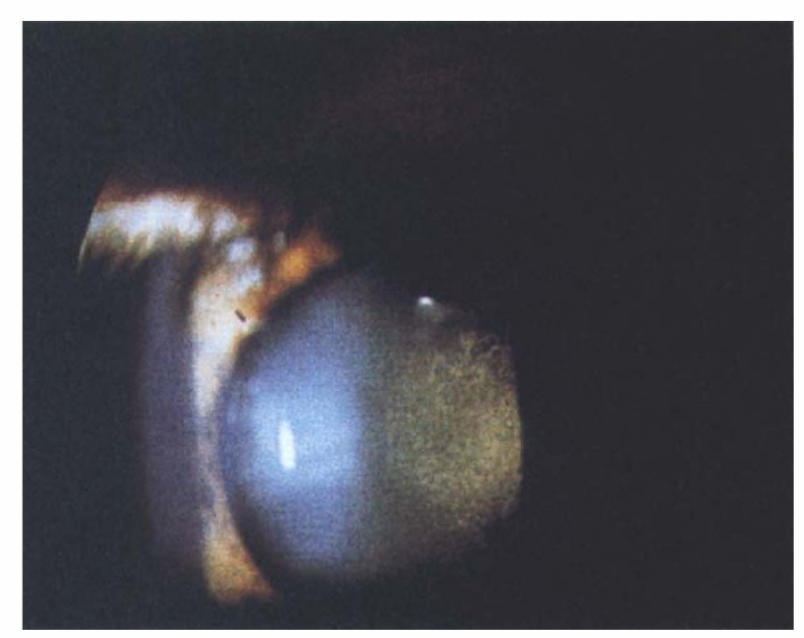

Fig. 1. A right posterior subcapsular cataract.

directly dependent upon voltage difference and inversely proportional to the electrical resistance. If the eye is interposed in this electrical circuit, the melanin granules of the pigment epithelium and the uvea constitute the main obstacle to the current flow. ${ }^{3}$ Hanna and Fraunfelder ${ }^{4}$ have shown that an electrical injury to the eye heats the pigmented portion of the iris, inducing lens protein denaturation and cataract formation. According to Duke Elder, ${ }^{5}$ an electric shock usually produces anterior subcapsular changes, whereas lightning strikes result in both anterior and posterior coarse subcapsular opacities. These opacities can impair vision significantly and often necessitate surgical removal. The prognosis for visual acuity after removal of associated cataracts is good $(6 / 6$ to $6 / 12){ }^{3}$ Other ocular injuries associated with lightning include punctate keratitis, iridocyclitis, papilloedema, retinal haemorrhage, retinal folds, retinal detachment, macular oedema and macular hole. ${ }^{2}$

\section{References}

1. Long JC. A clinical and experimental study of electric cataract. Trans Am Ophthalmol Soc 1962;60:471-516.

2. Noel LP, Clarke WN, Addison D. Ocular complications of lightning. J Pediatr Ophthalmol Strabismus 1980;17:245-6.

3. Jaffe GJ, Handa JT. Lightning maculopathy. Retina 1994;14:169-72.

4. Hanna C, Fraunfelder FT. Electric cataracts. II. Ultrastructural lens changes. Arch Ophthalmol 1972;87:184-91.

5. Duke Elder S. System of ophthalmology, vol 14, pt 2. London: H Kimpton, 1972:830.

S. Cazabon

T.R. Dabbs

Department of Ophthalmology

St James's University Hospital

Leeds, UK 
S. Cazabon

Department of Ophthalmology

St James's University Hospital

Beckett Street

Leeds LS9 7TF, UK

Tel: +44 (0)1132433144

e-mail: jcazabon@hotmail.com

Sir,

\section{Alkali injury complicated by choroidal neovascularisation}

Choroidal neovascularisation occurs in association with age-related macular degeneration, myopia, angioid streaks, ocular histoplasmosis and conditions associated with disruption of Bruch's membrane. It can occur without any associated ocular abnormality. Severe central visual loss occurs due to foveal involvement and macular scarring. The pathogenesis of choroidal neovascularisation is poorly understood. We report a case of an alkali injury which was complicated by the development of choroidal neovascularisation.

\section{Case report}

A 47-year-old Caucasian man presented to the Accident and Emergency department of the Birmingham and Midland Eye Centre. He had accidentally sprayed bleach to both eyes 3 hours previously. He had performed ocular irrigation immediately following the injury. He had previously had excellent visual acuity and was a low myope; his refraction was -2.50 DS in the right eye and -1.75 DS in the left eye. There was no history of previous ocular trauma. He was otherwise healthy. He had no history of foreign travel. There was no family history of ocular disease.

Immediate ocular irrigation was performed on arrival. The $\mathrm{pH}$ measured 8.00 in both eyes. Ocular examination revealed best corrected visual acuity was hand motions (HM) in the right eye and 6/18 in the left eye. Slit-lamp examination of the right eye revealed there was extensive epithelial loss affecting the cornea and conjunctiva, localised corneal oedema and limbal ischaemia extending for $240^{\circ}$, manifest by vascular occlusion. In the left eye there was mild corneal and conjunctival epithelial loss but no evidence of limbal ischaemia. Few fundal details could be discerned due to the anterior segment injuries. He was admitted for intensive treatment with topical corticosteroids, antibiotics, mydriatics, topical and systemic ascorbic acid.

Over the following 4 weeks, his visual acuity in the right eye failed to improve beyond $\mathrm{HM}$ and improved to $6 / 6$ in the left eye. The anterior segment injuries healed with the exception of symblepharon formation in the upper and lower fornices of the right eye (Fig. 1). Fundoscopy revealed a neurosensory detachment overlying the fovea, with retinal pigment epithelial changes and a grey subretinal lesion (Fig. 2a). There was no evidence of peripheral chorioretinal scarring, no

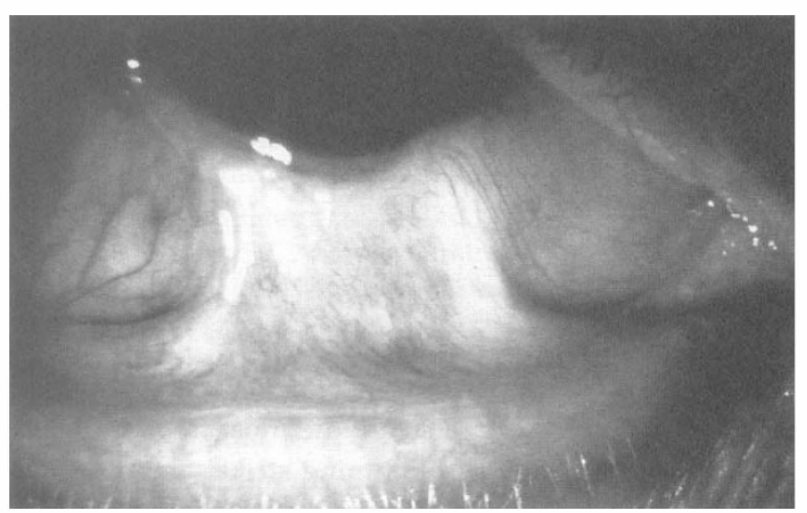

Fig. 1. Slit-lamp photograph of the right eye demonstrating conjunctival scarring and symblepharon formation inferiorly.

histoplasmosis spots and no peripapillary chorioretinal atrophy. Fluorescein angiography confirmed the presence of subfoveal classic choroidal neovascularisation (Fig. 2b, c). Due to the subfoveal location, it was judged that laser photocoagulation would not be of any benefit.

His acuity in the right eye has remained unchanged and he uses topical ocular lubricants to the right eye. His left eye is comfortable and normal.

\section{Comment}

Choroidal neovascularisation is a devastating cause of central visual loss. Ninety per cent of all patients with severe visual loss due to age-related macular degeneration have choroidal neovascularisation. The pathogenesis of choroidal neovascularisation is, however, poorly understood. Alteration to Bruch's membrane plays a key role in the development of choroidal neovascularisation. ${ }^{1}$

Multiple factors are required to induce choroidal neovascularisation. Factors are needed to cause degradation of the extracellular matrix and to stimulate growth of endothelial cells into blood vessels. Angiogenic factors necessary for the development of neovascularisation include vascular endothelial growth factor (VEGF) and fibroblast growth factor (FGF). ${ }^{2}$

The retinal pigment epithelium has been shown in vivo to produce angiogenic factors such as FGF and VEGF. ${ }^{3}$ Retinal pigment epithelium can be activated by a number of stimuli including hypoxia and laser photocoagulation. It has been shown that laser photocoagulation (associated with the development of choroidal neovascularisation) causes release of VEGF and FGF. ${ }^{4}$ It is not known why some eyes which undergo laser photocoagulation develop choroidal neovascularisation and others do not. Ischaemia may play a role, altering the balance between angiogenesis factors and angiogenesis inhibitors.

We report a patient who developed choroidal neovascularisation in association with an alkali injury. Alkali injuries cause ocular ischaemia. We believe that choroidal neovascularisation developed as a result of the 